\title{
Sales of Nonprescription Cold Remedies: A Unique Method of Influenza Surveillance
}

\author{
ROBERT C. WELLIVER, JAMES D. CHERRY, KENNETH M. BOYER, JAIME E. DESEDA-TOUS, \\ PETER J. KRAUSE, JAMES P. DUDLEY, ROBERT A. MURRAY, WILLIS WINGERT, JOHN G \\ CHAMPION, AND GERALD FREEMAN \\ Department of Pediatrics and Student Health Service, University of California, Los Angeles, Center for Health \\ Sciences, and Los Angeles County General Hospital and Public Health Department, Los Angeles, California. USA
}

\begin{abstract}
Summary
In 1976, the National Institute of Allergy and Infectious Disease sponsored a nationwide network for influenza surveillance. In this paper, in addition to reporting the surveillance findings in Los Angeles, sales of nonprescription cold remedies in a large supermarket chain were evaluated as an indicator of influenza activity in the community. Twenty-seven isolates of influenza B occurred between February 17 and April 26, 1977. Peak influenza B activity occurred from mid-March to early April, 1977. A 5-10\% increase in percent of respiratory and febrile respiratory illness seen in outpatient clinics was observed in late December and January. No variation in these statistics occurred during the peak of influenza activity. In contrast, sales of nonprescription cold remedies were apparently influenced by influenza B activity. Peak sales (345\% increase) occurred $4 \mathrm{wk}$ after the first influenza $B$ isolate and 1 wk before peak influenza activity was documented by peak rates of isolation.
\end{abstract}

\section{Speculation}

The data suggest that monitoring sales of nonprescription cold remedies may be a useful technique of influenza surveillance, especially in years when minimal activity occurs.

The standard method of influenza surveillance in the United States has been weekly monitoring of excess deaths due to pneumonia and influenza (4). However, significant changes in such statistics may not occur until as much as 1 month after the actual onset of epidemic influenza activity (6-8). Monitoring of school and industrial absenteeism may result in early detection of influenza activity, but such methods are limited because of increased holiday absenteeism in December and January (6-8). Marine et al. (5) recently demonstrated that the onset of influenza activity could be accurately detected through a year-round program in which viral surveillance cultures were routinely taken from children with respiratory illness. These authors confirmed a previous finding of Matlof et al. (6), that an increase in the percent of patients with respiratory illness seen in emergency rooms correlated with peak influenza activity in the community. Furthermore, with this surveillance system, antigenic drift of the prevalent virus strain could be detected several months before the occurrence of epidemic disease due to the new strain.

In 1976, the National Institute of Allergy and Infectious Disease sponsored a nationwide network for influenza surveillance because of the threat of a swine influenza epidemic. This program employed the method of Marine et al. In this paper, in addition to reporting the surveillance findings in Los Angeles, sales of nonprescription cold remedies in a large supermarket chain were evaluated as an indicator of influenza activity in the community. Influenza activity could not be detected using the statistical techniques of Marine et al. However, sales of nonprescription cold remedies accurately detected influenza activity, reaching a maximum at the onset of peak influenza activity in the community.

\section{MATERIALS AND METHODS \\ VIRAL SURVEILLANCE CULTURES}

Viral cultures were obtained from the first 20 children presenting with febrile respiratory illness each Thursday at the Los Angeles County Hospital Pediatric Clinic. Specimens were occasionally taken from similar patients presenting at the UCLA Student Health Service and Pediatric Outpatient Clinic. The program and procedures were explained and a signed statement of informed consent was obtained. Cultures were obtained by rotating cotton-tipped swabs on the pharynx. Swabs were then rinsed in small vials containing $4 \mathrm{ml}$ tryptose phosphate with $0.5 \%$ gelatin (Grand Island Biological Company, 3175 Staley Road. Grand Island, NY, 14072) and left in the vials while they were held at $4^{\circ} \mathrm{C}$ for $1-12 \mathrm{hr}$. Later, the swabs were removed and the contents of the vials were centrifuged. The supernatant was decanted and treated with $0.1 \mathrm{ml}$ of an antibiotic-antimycotic preparation (GIBCO). Aliquots of this solution were then inoculated onto monolayers of rhesus monkey kidney cells and human embryo lung fibroblasts (WI-38) (Flow Laboratories, $936 \mathrm{~W}$. Hyde Park Blvd., Inglewood, CA, 90302), chicken trachea organ cultures, and chorioallantoic and amniotic cavities of 10- to 11-dayold embryonated hen eggs (Pace Setters, Cucamonga, CA). Cultures were examined for cytopathic effect, decreased ciliary activity, or hemagglutination using standard methods $(1,2)$. Antisera used in testing were provided by the Center for Disease Control, Atlanta, GA.

\section{OUTPATIENT STATISTICS}

Outpatient statistics were obtained from the LA County-USC Medical Center, where a large volume of children are seen. Figures reflecting total visits, percent of respiratory visits, and percent of febrile respiratory visits were kept. Total visits refers to the number of outpatients seen each week. Percent of respiratory visits is the percent of children seen each Thursday with respiratory illness, whereas percent of febrile respiratory is the percent of respiratory visits in which there was an associated fever of $\geq 38^{\circ} \mathrm{C}$ or fever at home for which antipyretics were given. Respiratory illness was diagnosed in patients in whom the chief complaint or diagnosis was cough, congestion, rhinorrhea, tonsillitis, pharyngitis, croup, bronchitis, bronchiolitis, pneumonia, upper respiratory infection, "flu", or "cold."

\section{CALCULATED SALES OF ANTIPYRETICS AND NONPRESCRIPTION COLD REMEDIES}

Representatives of Ralph's Grocery Company, a large supermarket chain, provided computer printout sheets weekly which contained detailed information on quantities of different products 
on hand in a central distributorship. This central facility was responsible for stocking several supermarkets spread widely around Los Angeles. Satellite markets placed their orders with the central distributor on the basis of current sales, and these orders were filled generally within $72 \mathrm{hr}$. By comparing the quantities on hand in the central facility from week to week, an estimate of citywide sales of antipyretics and cold remedies was obtained. Results for each week are expressed as percent of increase or decrease in sales compared to a baseline figure, chosen as sales for the week ending November 18, 1976, which was the first week for which such information was available (Table 1).

The printout sheets also noted bargains or advertising campaigns designed to boost sales of individual products. Because intermittent deals or increased advertising could potentially influence sales of individual items, figures for several different brand names of the same compound were grouped. Separate figures were kept for adult antipyretics (Bayer aspirin, Bufferin, and Datril), children's aspirin (Bayer and St. Joseph's), and cold remedies (Contac, Coricidin, and Congesprin).

\section{RESULTS}

\section{VIRAL CULTURES}

The first influenza virus isolate was obtained from a 20 -yr-old college student in June 1976 and was characterized by the Center for Disease Control as an influenza A/Port Chalmers/73 strain. No further influenza $A$ viruses were recovered. Increased numbers of noninfluenza respiratory viruses (parainfluenza, rhinoviruses, and adenovirus) were recovered beginning in early January 1977. Influenza B was first isolated on February 17, 1977. Peak influenza B activity, determined by number of positive cultures, occurred from mid-March through early April 1977. During this 3-wkperiod, 16 of $49(33 \%)$ of all cultures taken were positive for influenza B (Fig. 1).

\section{OUTPATIENT STATISTICS}

As shown in Figure 2, the total number of outpatients seen each week varied considerably throughout the study period, and no correlation with number of positive viral cultures was observed. The percent of weekly visits due to respiratory illness was generally less than $25 \%$ until mid-December. From this point through the end of April, the percent of weekly respiratory visits varied between 30 and 35\% of total outpatient visits. Finally, $45-55 \%$ of all respiratory illness was associated with fever throughout most of the study period. From late January through the end of the study period, $55-65 \%$ of all respiratory illness was associated with fever.

\section{SALES OF ANTIPYRETICS AND NONPRESCRIPTION COLD REMEDIES}

Figure 3 shows the data for weekly sales of antipyretics and nonprescription cold remedies. Data on sales of these products became available to the authors in November 1976. Sales of adult and children's aspirin were generally within $\pm 25 \%$ of the initial figure and showed no variation with influenza B activity. Sales of cold remedies initially stabilized over a 4-wk-period at 63-95\% above the baseline figure. From late December through early March, sales were somewhat higher with values in the range of $71-166 \%$ above the baseline. Peak sales $(345 \%)$ occurred during the week ending March 17, 1977, coinciding with the onset of peak influenza activity. During the 3-wk-period of peak influenza activity, sales averaged $185 \%$ above the baseline figure. Neither

Table 1. Sales of antipyretics and cold remedies

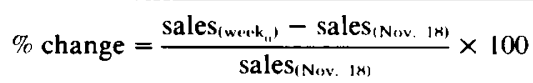

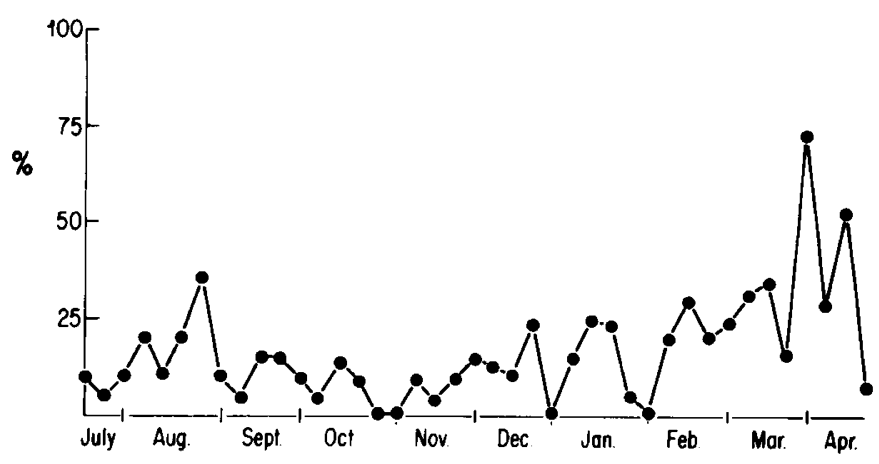

Fig. 1. Percent of cultures positive for respiratory viruses.

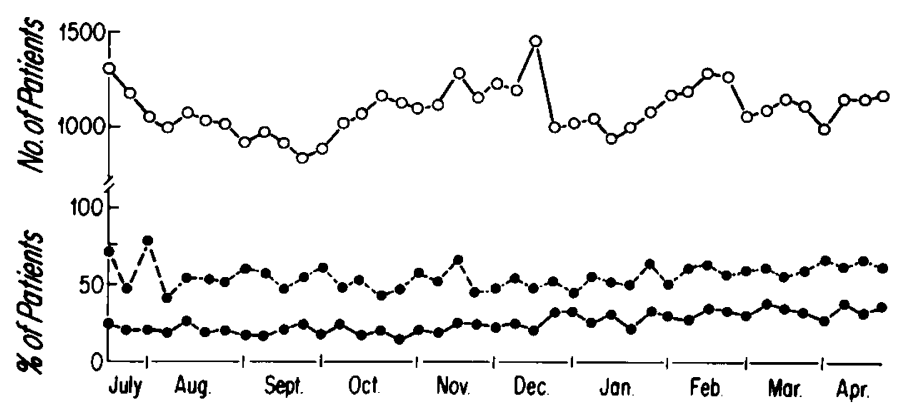

Fig. 2. Outpatient statistics $\mathrm{O}-\mathrm{O}-\mathrm{O}$ total weekly visits, with respiratory illness, ....... . . respiratory with fever.

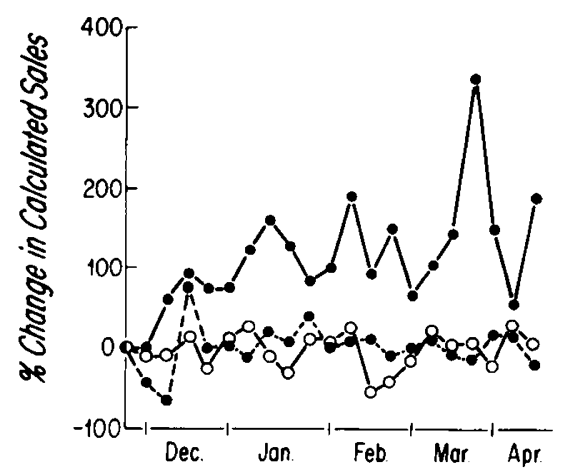

Fig. 3. Calculated sales of antipyretics and cold remedies children's aspirin, - - - - adult antipyretics, -10 cold remedies.

increased advertising nor offering of bargains occurred during the period of increased sales.

\section{DISCUSSION}

Peak influenza activity in Los Angeles in the winter of 19761977 occurred from mid-March through early April. During this period, influenza B was recovered from $33 \%$ of all cultures taken. A $5-10 \%$ increase in the number of patients seen with respiratory and febrile respiratory illness occurred earlier, in late December and January, concurrent with an increased rate of recovery of other, noninfluenzal respiratory pathogens. No variation in outpatient statistics occurred during the peak of influenza activity. In previous studies $(5,6,8)$ influenza epidemics were associated with isolation of influenza $\mathrm{A}$ in up to $75 \%$ of cultures taken. The $33 \%$ recovery rate in this study might suggest that influenza $B$ activity in the community was very mild. It is possible that outpatient statistical methods may detect only widespread epidemics typical of influenza $A$.

On the other hand, calculated sales of common cold remedies apparently were influenced by influenza B activity. Peak sales 
(345\% increase) occurred during the week ending March 17. This was $3 \mathrm{wk}$ after the first influenza $B$ isolate and $1 \mathrm{wk}$ before the onset of peak influenza activity. Sales averaged $185 \%$ above the baseline value during the period of maximum influenza activity (March 17 through April 7) compared to 100\% above the baseline for the first few months of 1977. No particular bargains or advertising campaigns could be related to this increase. In contrast, sales of adult and children's aspirin showed little variation throughout the study period. The data suggest that calculated sales of nonprescription cold remedies may be a sensitive indicator of influenza $B$ activity.

Monitoring of sales of nonprescription cold remedies has certain advantages over other methods of influenza surveillance. In comparison to routinely obtaining viral cultures, it is simple and inexpensive. In contrast to school and industrial absenteeism, sales of cold remedies apparently are not affected by the year-end holiday season. Finally, this method may be more sensitive than computation of outpatient statistics for detection of mild influenza epidemics. A potential disadvantage is that a modest increase in sales of nonprescription cold remedies was observed roughly simultaneously with an increased rate of recovery of noninfluenzal respiratory viruses (January 1977). The increase in sales at the time of peak influenza activity was much more pronounced, however.

An interesting fallout of the study was the recovery of an A/ Port Chalmers/73 strain from a patient with respiratory illness in June of 1976. The patient had no contact with laboratory personnel and A/Port Chalmers was not kept in stock in our laboratory, so infection was probably caused by a wild strain. Recovery of this strain several years after its epidemic activity was surprising because strains with considerable antigenic drift have subsequently become widespread (3).

\section{REFERENCES AND NOTES}

1. Chanock. R. M.: Parainfluenza viruses. In: E. H. Lennette and N. J. Schmidt: Diagnostic Procedures for Viral and Rickettsial Infections. 4th Edition p. 423 (American Public Health Association. Inc.. New York. 1969).

2. Cherry. J. D., and Taylor-Robinson. D.: Large quantity production of chicken embryo tracheal organ cultures and use in viral and mycoplasma studies. Appl. Microbiol., 19: 658 (1970).

3. Current trends: Influenza. Morbidity and Mortality Weekly Rep.. 25: 63 (1976).

4. Langmuir. A. D.. and Houseworth. J.: A critical evaluation of intluenza surveillance. Bull. WHO, 4I: 392 (1969).

5. Marine. W. M.. McGowan. J. E.. and Thomas. J. E.: Influenza detection: A prospective comparison of surveillance methods and analysis of isolates. Amer. J. Epidemiol., 102: 225 (1975).

6. Matlof. H., Murray, R. A.. Kami. I.. and Heidbreder. G. A.: Influenza in L.os Angeles County, 1968-69. HSMHA Health Reports, 86 : 183 (1971).

7. Rubin. R. J.. and Gregg. M. B.: Influenza surveillance in the United States. 1972-74. Amer. J. Epidemiol.. 102: 225 (1975).

8. Satz. J.. Prier. J. E. Riley. R.. Schrack. W. D.. Hrehorovich. V.. and Deardorff. J.: Epidemiological and biological characteristics of the Hong Kong influenza epidemic in Pennsylvania during 1968-1969. Amer. J. Public Health. 60: 2197 (1970).

9. The authors thank Connie Banks. secretary. and representatives of Ralph's Grocery Company. Los Angeles for providing sales information. The authors are also grateful for the technical assistance of Alice Garakian and (armen Castillo.

10. This research was supported by contract \#NOI-AI-625I0 National Institutes of Health and Allergy and Infectious Disease (NIH-NIAID)

11. Requests for reprints should be addressed to: James D. Cherry. Department of Pediatrics, UCLA Center for Health Sciences, 10833 LeC onte Avenue. Los Angeles. CA 90024 , USA.

12. Received for publication July 7. 1978.

13. Accepted for publication September 21. 1978. 